Jamshed Iqbal*, Mukhtar Ullah, Said Ghani Khan, Baizid Khelifa, and Saša Ćuković

\title{
Nonlinear control systems - A brief overview of historical and recent advances
}

https://doi.org/10.1515/nleng-2016-0077

Received June 7, 2016; accepted May 17, 2017.

\begin{abstract}
Last five decades witnessed remarkable developments in linear control systems and thus problems in this subject has been largely resolved. The scope of the present paper is beyond linear solutions. Modern technology demands sophisticated control laws to meet stringent design specifications thus highlighting the increasingly conspicuous position of nonlinear control systems, which is the topic of this paper. Historical role of analytical concepts in analysis and design of nonlinear control systems is briefly outlined. Recent advancements in these systems from applications perspective are examined with critical comments on associated challenges. It is anticipated that wider dissemination of this comprehensive review will stimulate more collaborations among the research community and contribute to further developments.
\end{abstract}

Keywords: Nonlinear control, Modern control, Challenges in control, Technological applications

\section{Introduction}

Real world systems are inherently nonlinear in nature at least when considered over wide operating range although many of the systems are assumed to 'behave' as linear in the vicinity of a certain operating point at low speeds under certain assumptions. Many physical processes are represented by nonlinear models. Examples include; coulomb friction, gravitational and electrostatic at-

\footnotetext{
*Corresponding Author: Jamshed Iqbal, Department of Electrical Engineering, FAST National University of Computer and Emerging Sciences, Islamabad, Pakistan, E-mail: jamshed.iqbal@nu.edu.pk Mukhtar Ullah, Department of Electrical Engineering, MY University, Islamabad, Pakistan

Said Ghani Khan, Department of Mechanical Engineering, College of Engineering Yanbu, Taibah University, Saudi Arabia

Baizid Khelifa, Department of Computer Science and Automatic Control, Mines-Telecom Institute, France

Saša Ćuković, Faculty of Engineering, University of Kragujevac, Kragujevac, Serbia
}

traction, voltage-current characteristics of most electronic systems and drag on a vehicle in motion. Recently, many researchers from such broad areas like process control, biomedical engineering, robotics, aircraft and spacecraft control have shown an active interest in the design and analysis of nonlinear control strategies. Thus, most of the real problems necessitate invariably bumping into nonlinearities [1].

Primary reasons behind growing interest in nonlinear control include [2]; improvement of linear control systems, analysis of hard nonlinearities, need to deal with model uncertainties and design simplicity. Nonlinear strategies improve trivial approaches by taking into accounts the dynamic forces like centripetal and Coriolis forces which vary in proportion to the square of the speed. So, the linear control laws put a serious constraint on speed of motion to achieve a specified accuracy. However, simple nonlinear controller can reasonably compensate the nonlinear forces thus achieving high speed in an ample workspace. Also, hard nonlinearities like dead-zones, hysteresis, Coulomb friction, stiction, backlash and saturation do not permit linear approximation of real-world systems [3]. After predicting these nonlinearities, nonlinear approaches properly compensate these to achieve unmatched performance. Moreover, real systems often exhibit uncertainties in the model parameters primarily due to sudden or slow change in the values of these parameters. A nonlinear controller through robustness or adaptability can handle the consequences due to model uncertainties [4]. The systems that are not linearly controllable or observable may be controllable/observable in a nonlinear sense.

Modern technology such as high accuracy high speed robots require fulfilling strict design requirements. The positioning of such robots is a nonlinear problem since it involves coordinate transformation matrices having sine and cosine terms. The linear control of the manipulators assumes each joint to be independent and considers the inertia 'seen' by each joint as a constant. This approximation leads to non-uniform damping throughout the workenvelope and results other undesirable effects [5]. Considering a 6 Degree Of Freedom (DOF) manipulator, the over performance of a nonlinear strategy particularly in the presence of a disturbance is evident from Fig. 1 where 
a bounded matched disturbance (Fig. 1a) is fed to the manipulator and tracking performance (Fig. 1b) of a nonlinear approach Sliding Mode Control (SMC) is compared with a linear strategy i.e. Computed Torque Control with Proportional Integral Derivative (CTC-PID).

Non applicability of superposition and homogeneity in case of nonlinear systems results in major implications on the analysis and design of the control systems [6]. The straight forward relationship between transfer function zero(s) and pole(s) locations and time response does not hold valid in general. An unforced nonlinear system can possess limit cycles not speculated by linear theory. The controllability and observability cannot be determined simply based on rank tests. Moreover, stability of a system is no longer just a simple function of eigenvalue locations since nonlinear systems may have multiple stable/unstable equilibrium points.

Owing to the pertinent importance of nonlinear control in wide range of recent applications, this paper presents a brief comment on the subject topic. Interested readers are encouraged to refer to the original literature cited for more specific details. The remaining paper is structured as follows: Section 2 briefly presents historical perspective of nonlinear control systems while recent advances and challenges are discussed in Section 3 and 4 respectively. Finally Section 5 comments on conclusion.

\section{Historical Perspective}

History of nonlinear control dates back to $18^{\text {th }}$ century when centrifugal "flyball" governor was invented to control steam engine by regulating the admission of steam into the cylinder. However, the governor was made to work without concrete analytical concepts [8].

In 1892, A.M. Lyapunov, a Russian mathematician, presented two methods in order to determine the stability of dynamic systems described by Ordinary Differential Equations (ODE). The second method called as direct method of Lyapunov, can determine stability without actually solving the ODE and thus finds potential in stability analysis of nonlinear control systems [9]. Lyapunov showed that if the linear approximation of a system is stable near an equilibrium point, then the truly nonlinear system will be stable for some neighborhood of that point. Lyapunov's work was translated into French in 1907 and recovered in control context later by Kalman and Bertram.

Other noticeable works which benefited nonlinear control include Van der Pol's research on electronic oscillations and Duffing's findings on nonlinear vibrations.

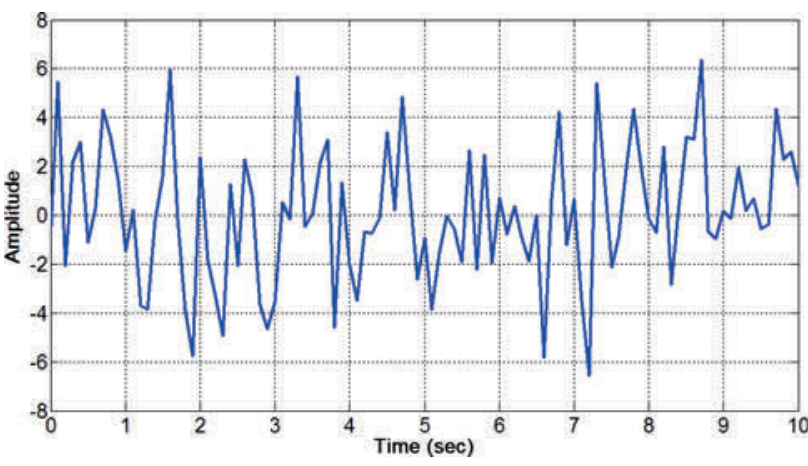

(a)

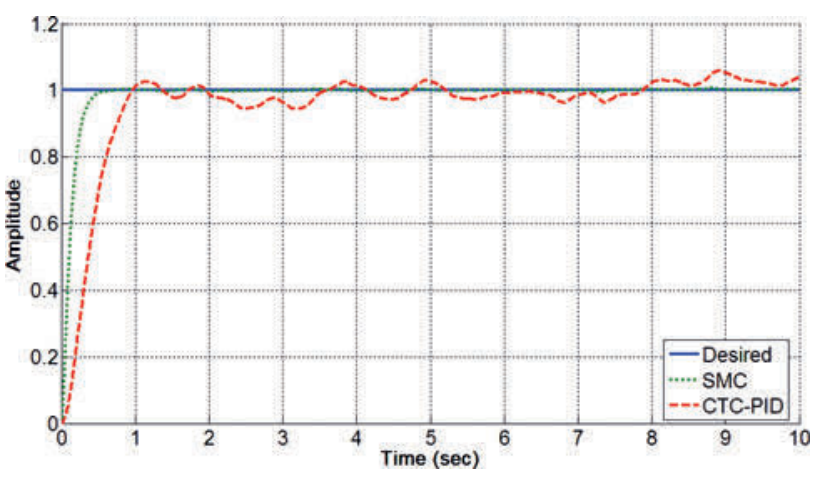

(b)

Fig. 1: Robustness comparison of linear and nonlinear control strategies [7] (a) Disturbance (b) Tracking performance

Based on the proposed nonlinear second order equations, approaches were developed to predict various phenomena of nonlinear systems, which include subharmonic oscillations, limit cycles, jump phenomena and frequency entrainment.

As a subject, control engineering was in its infancy till late 1930s when scientific community started to face the problem of servomechanisms control [10]. Such mechanisms, approximated by second order systems, were dealt with the phase plane method, introduced by Poincaré. The event of Second World War boosted research in nonlinear control of servomechanisms due to the functional requirements imposed by fire-control systems and control of guided vehicles. During 1940-1960, three main analytical approaches used for analyzing nonlinear systems include; the describing function, the phase plane method and various methods involving relay systems. In the classical era, most problems involved single input, single output, linear, finite dimensional and time-invariant systems.

Year 1960 is considered as start of modern era for nonlinear control [8]. This is the same time when the first International Federation of Automatic Control (IFAC) congress was held in Moscow, which brought theory of mechanics and Nyquist-Bode feedback theory closer to create to- 
day's control theory. The two key application drivers during this time were defense and space race. Other industrial avenues where nonlinear control were applied include automobiles, ships, steel, paper, minerals etc. The nonlinear, time varying, highly dimensional, poorly modelled and multivariable nature of the encountered real systems were outside the bounds of classical control theory. The digital computer was first introduced as a design tool and later as a component of a control system.

In early 1970s, scientists investigated that the notions of energy and dissipation are linked with Lyapunov theory. So, dynamic systems can be viewed as energy transformation mechanisms. Based on this concept, Willems Jan proposed a theory for dissipative systems [11]. In 1990s, Sontag and Wang proposed theory of input-to-state stability for nonlinear control systems [12], which can analyze stability of complex structures based on behavior of elementary subsystems and has been successfully applied to biological and chemical processes [13]. In 1995, Isidori presented geometric control theory by introducing the concept of zero dynamics [14]. With the ability to analyze controllability and observability, differential geometry finds enormous potential in the domain of nonlinear control systems.

A critical review of early history of nonlinear control shows that concepts related with optimality, stability and uncertainty were descriptive rather than constructive. 1990s is considered as decade of 'activation process' when the descriptive research was being transformed into formal design methods through constructive procedures [15]. Table 1 summarizes prominent historical advances which directly or indirectly enriched the domain of nonlinear control systems.

\section{Mathematical Viewpoint}

Developments in pure and applied Mathematics and to some extent in Physics have a great role in evolving nonlinear control strategies. Application of Differential algebra and multivariable calculus for understanding, formulation and conceptual solutions to the problems in automatic control resulted in various nonlinear control strategies. Detailed reviews of these strategies are reported in [5, 40].

As an educational example, a variable structure control technique, SMC is selected here to be examined from mathematical perspective due to its robustness feature and long history of theoretical and practical developments. This control technique has now become a de-facto
Table 1: Historical overview of advances in nonlinear control

\begin{tabular}{|c|c|c|}
\hline Year & Work of & Contribution [Ref.] \\
\hline $1880 s-90 s$ & Poincare & $\begin{array}{l}\text { - Study of nonlinear dynam- } \\
\text { ics as } n \text {-body problem in ce- } \\
\text { lestial mechanics [16] } \\
\text { - Concept of limit cycles and } \\
\text { bifurcation theory [17] }\end{array}$ \\
\hline 1892 & Lyapunov & $\begin{array}{l}\text { Stability analysis of dynamic } \\
\text { systems [18] }\end{array}$ \\
\hline $1910 \mathrm{~s}$ & Duffing & $\begin{array}{l}\text { Modelling a dynamic chaotic } \\
\text { system using } 2 \text { nd order non- } \\
\text { linear differential Eq. [19] }\end{array}$ \\
\hline $1920 s$ & Van der Pol & $\begin{array}{l}\text { Study of limit cycles in re- } \\
\text { lation with oscillator dynam- } \\
\text { ics [20] }\end{array}$ \\
\hline $1930 \mathrm{~s}$ & Bode & $\begin{array}{l}\text { Asymptotic representation of } \\
\text { the frequency response [21] }\end{array}$ \\
\hline $1930 \mathrm{~s}$ & Krylov and Bogoliubov & $\begin{array}{l}\text { - Existence of invariant mea- } \\
\text { sures theorem [22] } \\
\text { - Describing function method }\end{array}$ \\
\hline 1932 & Nyquist & $\begin{array}{l}\text { Regeneration (or Feedback) } \\
\text { theory [23] }\end{array}$ \\
\hline 1944 & Lur'e & $\begin{array}{l}\text { Abolsute stability prob- } \\
\text { lem [24] }\end{array}$ \\
\hline $1950 \mathrm{~s}$ & Emelyanov & $\begin{array}{l}\text { Theory of variable structure } \\
\text { system with sliding mode } \\
\text { control [25] }\end{array}$ \\
\hline 1960 & \multicolumn{2}{|c|}{ - Start of modern era for nonlinear control } \\
\hline $1960 s$ & Edward Norton Lorenz & $\begin{array}{l}\text { - Chaos theory [26] } \\
\text { - Butterfly effect [27] }\end{array}$ \\
\hline 1960 & Kalman and Bertram & $\begin{array}{l}\text { Recovered Lyapunov's work } \\
\text { in control context [28] }\end{array}$ \\
\hline 1961 & Popov & $\begin{array}{l}\text { - Cicle criterion for asymp- } \\
\text { totic stability [29] } \\
\text { - Concept of hyperstability }\end{array}$ \\
\hline 1962 & Yakubovich & $\begin{array}{l}\text { Connections b/w Lur'e and } \\
\text { Popov's results [30] }\end{array}$ \\
\hline 1970 & Various scientists & $\begin{array}{l}\text { Viewing dynamic systems as } \\
\text { energy transformation mech- } \\
\text { anisms }[31,32]\end{array}$ \\
\hline 1971 & Luenberger & $\begin{array}{l}\text { Concept and construction of } \\
\text { state observer [33] }\end{array}$ \\
\hline 1972 & Willems Jan & $\begin{array}{l}\text { Theory for dissipative sys- } \\
\text { tems [34] }\end{array}$ \\
\hline 1978 & Richalet et al. & $\begin{array}{l}\text { Model predictive heuristic } \\
\text { control [35] }\end{array}$ \\
\hline $1980 \mathrm{~s}$ & Zames & $\begin{array}{l}\text { Formulation of } \mathrm{H} \infty \text { con- } \\
\text { trol [36] }\end{array}$ \\
\hline 1989 & Ortega and Spong & $\begin{array}{l}\text { Concept of Passivity Based } \\
\text { Control (PBC) [37] }\end{array}$ \\
\hline 1990 & - Start of 'activation pro & cess' \\
\hline 1990s & Sontag and Wang & $\begin{array}{l}\text { Theory of input-to-state } \\
\text { stability for nonlinear sys- } \\
\text { tems [38] }\end{array}$ \\
\hline 1995 & Isidori & Geometric control theory [39] \\
\hline
\end{tabular}


solution to handle modeling and parametric uncertainties of a nonlinear system. Its other distinguishing features are reduced-order compensated dynamics and finite-time convergence.

The core idea behind SMC is to drive the nonlinear dynamics of the plant onto the selected sliding surface (reaching phase). The dynamics is then maintained at this surface for all subsequent time irrespective of nonlinearities. Figure 2 conceptualizes this concept.

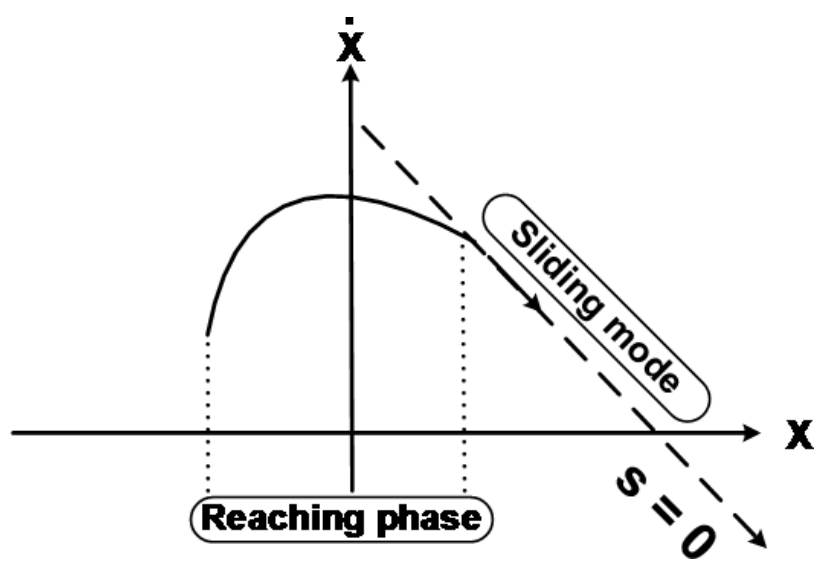

Fig. 2: Graphical representation of SMC

Considering a general architecture [7] with a nonlinear system assumed to be in canonical form i.e.

$$
\dot{x}=g(x, t)+a(x, t) u+\Delta(x t)
$$

where $u \in R$ is the control input and $x \in R^{n}$ is the vector corresponding to the measurable states of the system. $g(x t)$ and $a(x, t)$ represent smooth vector fields and are known terms. $\Delta(x t)$ representing matched uncertainties is assumed to be norm bounded i.e., $\Delta(x, t)=a(x, t) \delta(t) . \delta(t)$ is known and satisfies $\|\delta(t)\| \leq K^{\star}$ where $\mathrm{K}$ is a positive constant.

To achieve the objective of directing the states to zero even in the presence of disturbances, we define a sliding surface as

$$
s=C x(t)
$$

where $C$ is a row vector of the dimensions same as that of the states vector. The choice of elements of $C$ ensures that $s$ becomes Hurwitz monic polynomial. The control law keeps $s=0$, which results in order reduction by evolving the system with $n-1$ states in sliding mode. This ultimately makes the feedback system insensitive to the matched disturbances.

The control law for SMC consists of a nominal feedback control term and an additional part to deal with un- certainties. The overall control law can be written as

$$
u=u_{e q v}+u_{d i s c}
$$

where $u_{\text {eqv }}$ corresponds to the equivalent control input and $u_{\text {disc }}$ is a discontinuous control term. The design of $u_{\text {eqv }}$ involves posing $\dot{s}=0$ along the dynamics of (1) while assuming that $\Delta(x, t)=0$. Therefore,

$$
u_{e q v}=a^{-1}(x, t)(-g(x t))
$$

This control law is capable of ensuring that $\dot{s}=0$. However, the condition $s \neq 0$ may occur demonstrating that the sliding mode is not in practice. Thus, $u_{\text {disc }}$ is designed so as to mitigate the consequences of disturbances finally maintaining $s=0$. This term can be designed based on a Lyapunov function of the form

$$
v=\frac{1}{2} s^{2}
$$

Differentiating w.r.t. time along (1), we get

$$
\dot{v}=s\left(\frac{\partial s}{\partial x}(g(x, t)+a(x, t) u+\Delta(x, t))\right)
$$

or

$$
\dot{v}=s\left(a(x, t) u_{d i s c}+\Delta(x, t)\right)
$$

Using norm bounded assumption, (5) reduces to

$$
\dot{v}=a(x, t) s\left(u_{d i s c}+\delta(t)\right)
$$

Considering $u_{\text {disc }}=-K \operatorname{sign}(s)$, (6) becomes

$$
\dot{v}=a(x, t)(-s \operatorname{Ksign}(s)+s \delta(t))
$$

or

$$
\dot{v} \leq-|| a(x, t)|||s|(K-|\delta(t)|)
$$

Therefore,

$$
\dot{v} \leq-\mu|s|=-\sqrt{2} \mu v^{\frac{1}{2}}
$$

provided that $K-|\delta(t)| \geq \mu>0$. The inequality in (7) establishes that that sliding mode takes place in finite time even in the presence of uncertainties. Consequently, the closed loop dynamics can be written as

$$
\left[\begin{array}{c}
\dot{x}_{1} \\
\dot{x}_{2} \\
\vdots \\
\dot{x}_{n-1}
\end{array}\right]=\left[\begin{array}{ccccc}
0 & 1 & 0 & \ldots & 0 \\
0 & 0 & 1 & \ldots & 0 \\
\vdots & \ldots & \ddots & 1 & 0 \\
-c_{1} & -c_{2} & \ldots & \ddots & -c_{n-1}
\end{array}\right]\left[\begin{array}{c}
x_{1} \\
x_{2} \\
\vdots \\
x_{n-1}
\end{array}\right]
$$

Choice of positive values of the constants $c_{i}$ in (2) ensures that the poles of the feedback system are in Left Half 
Plane (LHP). As a final comment, $x \rightarrow 0$ as $t \rightarrow \infty$ and the system in (8) is not influenced by the uncertainties highlighting the invariance feature of SMC.

An ideal SMC may require infinitely fast switching in an attempt to accurately track the reference trajectory. However, practical switched controllers have imperfections limiting the switching frequency. Thus the representative point may oscillate around the selected sliding surface leading to an undesirable phenomenon termed as chattering. Figure 3 illustrates this concept. Solutions to this problem are discussed in [41].

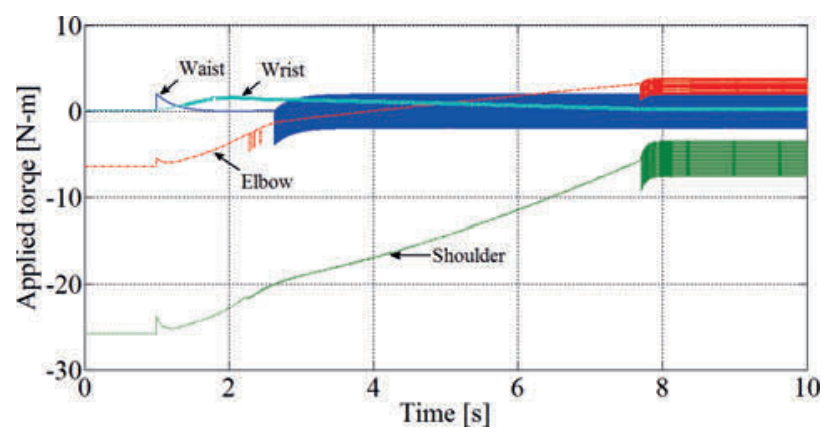

Fig. 3: Joints' torques resulting from simultaneous motion of six DOF robotic manipulator [42]

\section{Recent Advances}

In the last two decades, the advancements in nonlinear control systems have been in two folds; advances in theoretical approaches and more importantly applicationdriven developments. In theory, major breakthrough has been seen in the areas of sliding control, feedback linearization and nonlinear adaptive strategies. Recently, nonlinear control systems have gained high popularity primarily due to the extensive application of theoretical concepts to solve real world problems in various domains like electrical, mechanical, medical, avionics, space etc. Moreover, the advances in computer hardware and information technology have greatly resolved the computational constraints on analysis and design of nonlinear control systems. Now we see numerous applications of nonlinear control systems ranging from 'drive by wire' cars to 'fly by wire' aircraft flight control systems, to robotic, medical, industrial and space systems.

Robotic manipulators have reshaped the industrial automation and are now an integral part of most of the modern plants. Although linear control strategies like PID [43] have been the main workhorse in industry since decades, however, the trend to employ nonlinear approaches is gradually increasing [44]. A typical example of implementation of a nonlinear approach i.e. SMC on a customdeveloped pseudo-industrial platform [45] is presented in [42]. The control objective was to ensure tracking of desired trajectory $\left(q_{d}\right)$. This was achieved by defining a sliding manifold $(\mathrm{S}=\dot{e}+\mathrm{Ce})$ based on error signal $\left(e=q_{d}-q\right)$. The control law giving required input joint torque $(\tau)$ is expressed in (9) while the corresponding blockdigram is illustrated in Fig. 4.

$$
\tau=\mathrm{M}(\mathrm{q}, \dot{q})\left(\ddot{q}_{\mathrm{d}}-\mathrm{C} \dot{e}\right)+\mathrm{V}(\mathrm{q}, \dot{q})+\mathrm{G}(\mathrm{q})-\mathrm{K} \operatorname{sgn}(\mathrm{C} \mathrm{e}+\dot{e})
$$

where $M(q)$ denotes the inertia matrix, $V(q, \dot{q})$ is the matrix comprising of Centrifugal and Corollis forces and $G(q)$ represents the gravity effect. The matrices $\mathrm{K}=\operatorname{diag}\left\{\mathrm{k}_{1} \mathrm{k}_{2} \mathrm{k}_{3} \mathrm{k}_{4}\right\}$ and $\mathrm{C}=\operatorname{diag}\left\{\begin{array}{lll}\mathrm{c}_{1} \mathrm{c}_{2} & \mathrm{c}_{3} \mathrm{c}_{4}\end{array}\right\}$ are sliding surface constant and switching gain constant respectively. The system dynamics can be altered by varying $K$ and $C$.

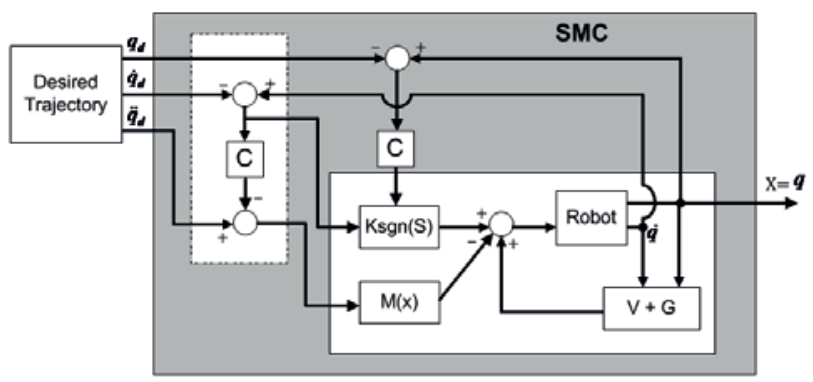

Fig. 4: Block diagram of SMC [46]

A more advanced variant of SMC, Integral Sliding Mode Control (ISMC) eliminates reaching phase and improves performance in terms of position error. It also significantly reduces the chattering phenomena. Based on ISMC law, Khan and Jalani proposed and experimentally validated a scheme for a humanoid robot arm control. They have also demonstrated compliance control via this scheme employing joint torque sensors [47]. The scheme is illustrated in Fig. 5. Recently, the book by Speirs et al. [48] presents bio-inspired nonlinear control for generating human-like posture, mimicking human motion and model reference adaptive compliance approaches. They have addressed the associated actuator saturation issues by introducing anti-windup compensators.

In medical domain, recent applications of nonlinear control includes anesthesia administration and control of devices for rehabilitation and prosthetics [49-51]. A nonlinear control approach to regulate Depth Of hypnosis (DOH) in Propofol anesthesia is reported in [52]. The pa- 


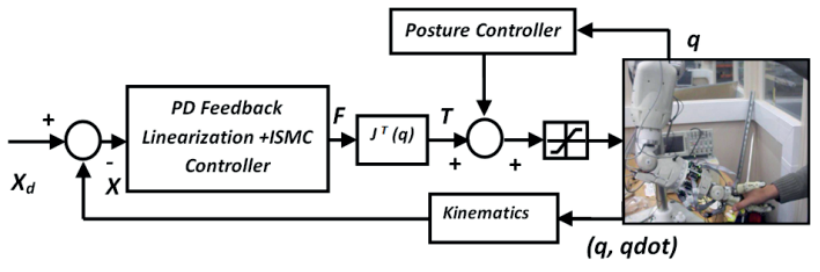

Fig. 5: ISMC scheme for humanoid robotic arm [47]

tient's hypnosis level is measured by EEG-based sensors e.g. Bispectral Index (BIS). Based on the derived patient's model, SMC minimizes steady state error to maintain hypnosis level within allowable range so as to conduct surgical procedures. Fig. 6 presents simulation results of eight patients after the infusion of drug for surgery. DOH level of 100 represents awake state while the level of 40-60 refers to moderate hypnotic state and is considered as safe range to execute surgery. As shown in the figure, all the patients achieved the desired level of hypnosis.

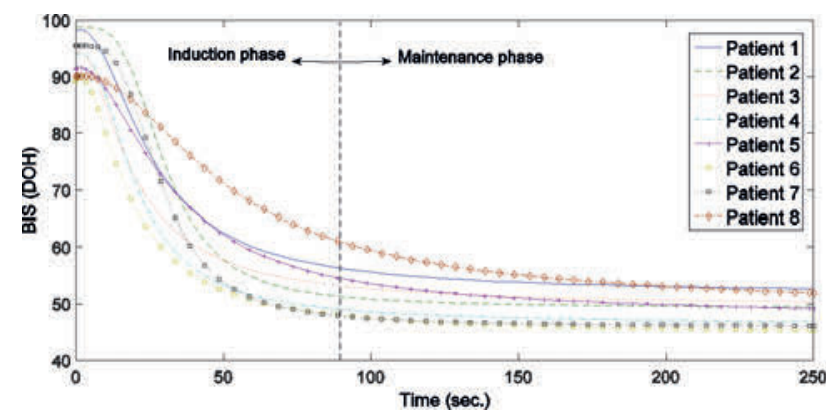

Fig. 6: Performance of nonlinear controller - Results show that there is no overdose of anesthesia [52]

The last decade has seen the emergence of the systems biology approach to understand biological systems in a holistic manner [53]. Rather than enumerating individual components (molecules, proteins) the systems biology approach focuses on the interactions between subsystems in order to understand the emergent dynamic behavior of the living system. Both the structural and functional organizations are important for characterizing the so-called symbiotic state of the biological system under study [54]. The structural organization involves network topologies including gene regulatory networks and biochemical reaction networks, and physical layers including molecules within organelles, organelles within cells, cells within tissues, tissues with organs and so forth. The functional organization involves cell functions including cell growth, cell division, cell differentiation (specialization) and cell death (apoptosis). The essence of systems biology is an understanding of the nonlinear dynamics of the biological system, which in turn requires the developments of computational models. Construction and analysis of such models of different subsystems (modules) of a system allow us to identify feedback loops in the system. Computer simulations could be used to test a drug or therapy before expensive clinical trials. In control-theoretic terms, a disease could be represented by some region of the state space of a living system. Viewed as a control system, the reference or desired state of the system could represent the healthy state. The drug or therapy could be represented by a controller in the loop. An application of nonlinear control can be found in [55] where the authors apply feedback linearization and optimal control strategies to a nonlinear state-space model of HIV infection. Other applications of optimal control of biological systems can be found in [56]. The curious reader might wonder why nonlinearity is ubiquitous in biological systems. This question can be answered fully in a brief account like this text but a justification will be provided by a few examples. Under appropriate assumptions, the mass action kinetics and mass action-like kinetics. Mass action-like kinetics are obeyed by intracellular biochemical reactions, cell-cell interactions and inter-species interactions including the epidemiological and predator-prey interactions. The state variables in mass action-like kinetics multiply which gives rise to nonlinear terms in the differential equations [53]. For instance, the rate of a chemical interaction between two species, commonly written as

$$
A+B \stackrel{k}{\longrightarrow} C
$$

depends on the probability of encounter (collision) per unit time of the reactant (interacting) species. Computing such a probability involves multiplication of the abundances of the reactant species. Other sources of nonlinearity include the feedback mechanisms in which a product of a reaction cascade could enhance or inhibit the reactants. Simplifications resulting from specific assumptions (e.g. quasi-steady state) including the Michaelis-Menten kinetics and Hill kinetics are in the form of fractions, and hence nonlinear terms in the model. The rich variety of such rateequation models has been outlined in Fig. 7 .

In chemical industry, the control of basic variables like pressure, levels, temperatures, flows and some quality variables is usually achieved by PID based law enhanced with advanced structures such as feedforward control, cascade control, ratio control, dead-time compensators etc. Common process characteristics in a chemical industry include [57]; unmeasured state variables and disturbances, multivariable interactions between variables, high order and distributed processes, uncertain and timevarying parameters and dead-time on inputs and measure- 


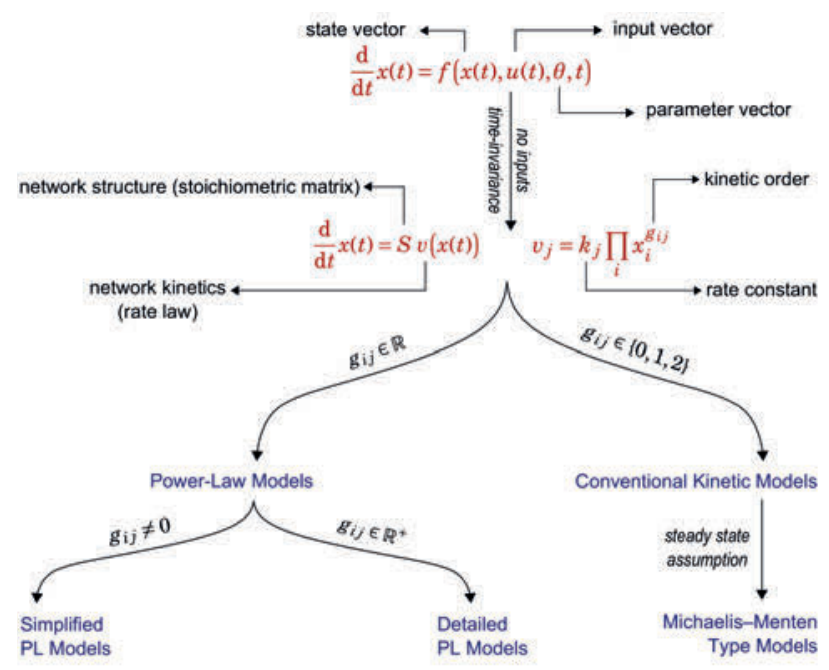

Fig. 7: Variety of rate-equation models of chemical reaction networks arising in systems biology [52]

ments. Complex problems arising due to constraints, delays, lags and model uncertainty can better be resolved using nonlinear control approaches. Owing to these reasons, Model Predictive Control (MPC), explicitly based on nonlinear dynamic representation of the chemical process, was developed. MPC is based on mathematical optimization and is now de-facto standard in nonlinear control of process industries. Other related concepts include Dynamic Programming (DP) and iterative learning control for batch processes. A new trend [58] is to realize MPC law based on Support Vector Machine (SVM) and Genetic Algorithm (GA) for trajectory tracking and disturbance rejection. Nonlinear control techniques are gradually replacing their linear counterparts in refineries and petrochemical plants to handle chemicals and polymers [59]. A detailed review presenting challenges and progress of control systems in chemical industry is reported in [60].

Most of the power systems involve varying operating conditions and/or uncertainties, some of which may not be anticipated a priori [61]. Power systems e.g. Flexible AC Transmission Systems (FACTS) demand controllers that have to tuned online, thus making the trivial decentralised control laws inadequate. The major contributions of nonlinear control in power systems is to; regulate frequency and voltage, adequately damp the oscillations and preserve synchronisation in the presence of disturbances. Relevant techniques with eminent recent advances include; Nonlinear PI [62], Optimal Control [63], MPC [64], Passivity-based control [65], SMC [66] and Observer-based SMC [67].

In power electronics, commonly encountered circuits exhibit nonlinear dynamics primarily due to the conse- quences of cyclic switching [68]. Existence of chaos, bifurcations and limit cycles in the circuits highlights the governing role of nonlinear control. In this context, MPC and SMC are common in power electronics owing to its ability to handle system constraints, multi-variable case and nonlinearities. Applications of MPC with prominent works in power electronics has been systematically reviewed in [69]. Scientific literature reports several successful implementations of control laws for applications like Uninterruptible Power Supplies (UPS), Active Front End (AFE), drives for induction machines and power converters connected to RL loads. Noticeable recent advancements include; SMC [70] and its variants [71, 72], Observer-based control [73] and reinforcement learning based nonlinear control [74]. Further control approaches based on physical principles e.g. current-limitation capability [75] and capacitor ampere-second balance [76] have also been proposed.

Other recent applications of nonlinear control systems are reported for vehicle state estimation [77] and for control of Unmanned Aerial Vehicles (UAV) [78], Autonomous Underwater Vehicle (AUV) [79], Nuclear steam generator [80], reactor [81], building's heating and cooling [82], power pricing and management [83] and so on.

Another trend which is gaining popularity since last two decades is to introduce adaptability and optimality in traditional nonlinear control strategies.

\subsection{Nonlinear adaptive control}

The most effective and advanced nonlinear control schemes are the adaptive controllers. The basic idea of an adaptive controller is to estimate uncertain parameters and to tune the controller online to adopt to the dynamic situation where the variations in the system parameters or environment are significant. Adaptive control got more popularity in 1950s in the aerospace control community. However, due to lack of well-understood stability tools and proper hardware, it lost its charm. Another particular reason for this loss of interest was incident of the plane crash which was controlled by an adaptive law [84]. Nevertheless, interest in adaptive control has revived especially in social robotic because of its suitability for a highly nonlinear, unstructured and dynamic human environment as compared to the controlled and well-ordered industrial work cells.

In general, there are two main types of adaptive control schemes i.e. Model Reference Adaptive Control (MRAC) (see Fig. 8) and Self Tuning Adaptive Control (STAC) (Fig. 9). In MRAC, the adaptation mechanism estimates suitable parameters, so that the system behave the 
same as the reference model. In STAC, the slowly varying parameters or unknown constants are optimized so as to minimize/ maximize an objective function typically minimization of error or maximization of efficiency.

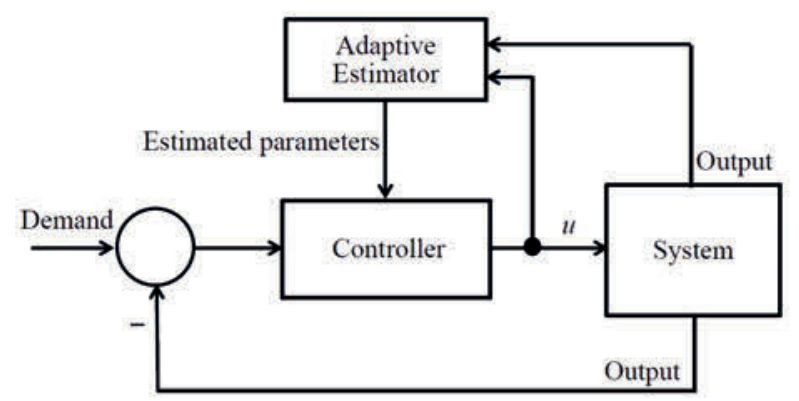

Fig. 8: Model reference adaptive control [85]

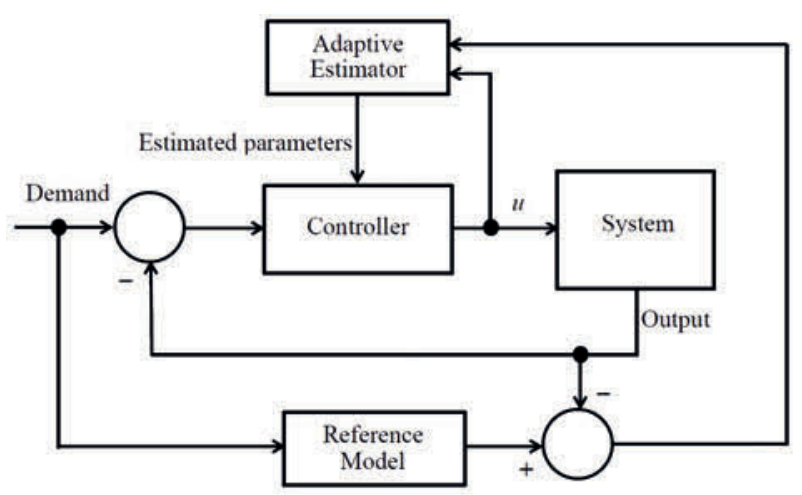

Fig. 9: Self tuning adaptive control [85]

Adaptive controllers may employ dynamic model and estimate the uncertain parameters [86] or may be dynamic model free. Model based adaptive controllers can be easily tuned and their performance is usually better as observed be Khan et al. [85]. However, dynamic model becomes very cumbersome as the DOF increases. This may results in computational and communication issues thus making the implementation of dynamic model based adaptive schemes more challenging. Therefore, dynamic model free adaptive controllers can be the best choice for a high DOF plant, eliminating the need for a dynamic model.

\subsection{Nonlinear optimal control}

The most effective and advanced nonlinear control adaptive controllers are very powerful in terms of tracking accuracy and fast response. However, adaptive control action usually involves high energy and aggressiveness. This may be a major disadvantage in application where optimal usage of resources is critical e.g UAV drones and autonomous robots. On the other hand, traditional linear optimal control techniques e.g. LQG and $\mathrm{H} \infty$ are offline techniques and require very accurate model of the system as well as full state feedback.

Reinforcement learning is one of the key methods to introduce optimality into the adaptive control schemes. Sutton et al. [87] have argued that reinforcement learning based adaptive optimal schemes are direct adaptive controllers. In direct adaptive controllers, the estimated parameters are employed directly to calculate the control signal where as in indirect methods, the estimated values are used in finding controller's parameters.

Reinforcement learning based optimal adaptive controllers solve nonlinear algebraic Riccati equation online. It usually consists of an Actor-Critic structure as shown in Fig. 10. Critic evaluates the action taken by the actor i.e. rewarding it (or punishing it). Subsequently, the control policy is updated and finally developing an optimal control policy over time.

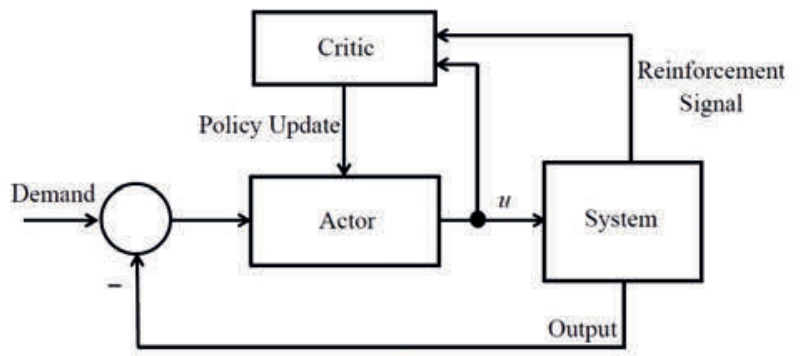

Fig. 10: Actor-Critic Structure for reinforcement learning scheme [88]

\section{Tutorial Example}

As an educational example, an inverted pendulum mounted on a moving cart is presented in this section. Fig. 11 depicts a cart of (effective) mass $M$ pivotted with an inverted pendulum of mass $m$ and (massless) rod length $l$.

The system is driven by a horizontal force $u$ applied at the cart. The two outputs include the (horizontal) cart displacement $x$ and the pendulum angle $\varphi$. Assume viscous friction of coefficient $b$. Application of basic Newtonian methods, or the more advanced energy methods based on Lagrangian, leads to following pair of nonlinear ordinary 


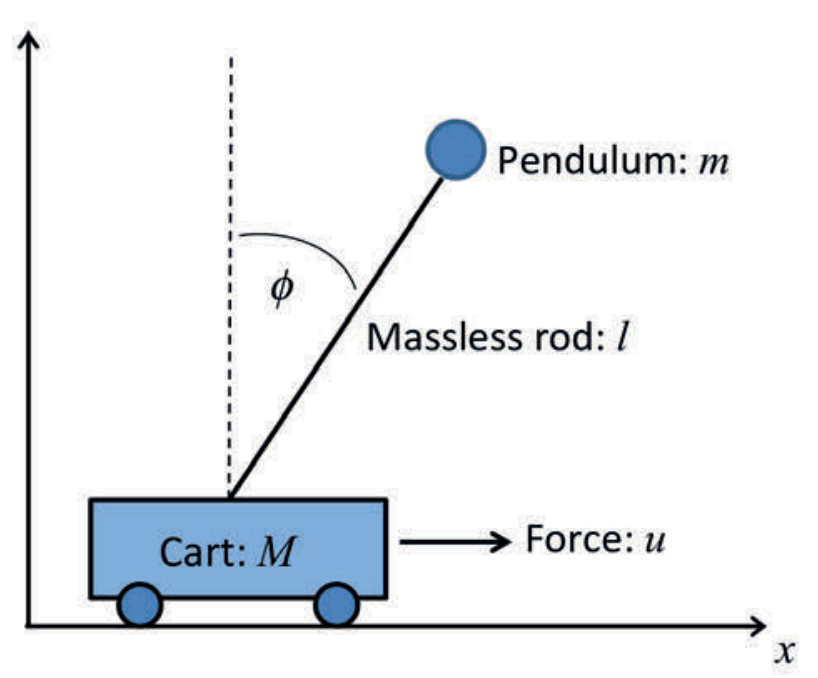

Fig. 11: Cart with an inverted pendulum

differential equations

$$
\begin{aligned}
l \ddot{\phi}+\ddot{x} \cos \varphi= & g \sin \varphi(m+M) \ddot{x}+b \dot{x} \\
& +m l\left(\ddot{\phi} \cos \varphi-\dot{\varphi}^{2} \sin \varphi\right)=u
\end{aligned}
$$

The nonlinearity arises from the trigonometric functions, products, and squares of the outputs. It is often useful to rewrite such equations into a set of first-order differential equations that is referred as state-space form:

$$
\dot{z}=f(z, u)
$$

where $z$ is a vector of state variables and $f$ is a vector of functions. For the system at hand, once chouce of state variables could be

$$
z_{1}=\varphi, z_{2}=\dot{\varphi}, z_{3}=x, z_{4}=\dot{x}
$$

which will require $f_{1}=\dot{\varphi}$ and $f_{3}=\dot{x}$. The other two functions $f_{2}$ and $f_{4}$ could be worked out algebraically. The nonlinear state-space model can be linearized around a suitable equilibrium, say the origin of the 4-dimensional state space. The resulting linear system would be of the form

$$
\delta x=A \delta x+B \delta u
$$

where $\delta x$ is the deviation of the state vector $x$ around the equilibrium state and $\delta u$ is the deviation of the input $u$ around the specific input required to maintain the equilibrium state. For the model at hand, the coefficient matrices can be worked out to

$$
A=\left[\begin{array}{cccc}
0 & 1 & 0 & 0 \\
\frac{g(M+m)}{I M} & 0 & 0 & \frac{b}{T M} \\
0 & 0 & 0 & \\
-\frac{m g}{M} & 0 & 0 & -\frac{b}{M}
\end{array}\right], B=\left[\begin{array}{c}
0 \\
-\frac{1}{I M} \\
0 \\
\frac{1}{M}
\end{array}\right]
$$

The reader is encouraged to compute the eignevalues of $A$ for the following parameters: $M=3 \mathrm{~kg}, m=0.2 \mathrm{~kg}, \mathrm{l}=0.31 \mathrm{~m}$, and $b=0.1 \mathrm{Ns} / \mathrm{m}$. It should turn out that some of the eigenvalues have positive real parts. That corresponds to an unstable system. The eigenvalues, also called system poles, can be relocated by MATLAB commands acker and place. Such a relocation is useful not only to stablize a system but also to achieve desired closed-loop behavior such as good damping and a fast response.

\section{Challenges}

Nature is nonlinear and the best way will be to deal with it with nonlinear approaches. Although, linear control has been successfully employed for decades. The problem with linear system is that it may not be able to cater for the modern and advanced technology such as multi-degree of freedom robots (with under actuation or constraints), UAVS, submarines and missile control in which more complex system operations are involved and greater application ranges are required.

The decision to choose linear or nonlinear control for a particular application may be a difficult one. Linear control is well tested and the people in industry have confidence in it. There are plenty of excellent analysis tools available for linear systems such as Bode plot, root locus, Nyquist stability criteria, Laplace transform, Z-transform and Fourier transform etc. In contrast, nonlinear system needs complex mathematical analysis e.g. Lypunov stability criterion, Popov criterion and singular perturbation methods. Mathematical modelling may also be cumbersome for nonlinear systems. Nonlinear system may suffer from limit cycle, chaos and bifurcation. Most of the schemes can only ensure local stability while global stabilities cannot be guaranteed [2].

In addition, computational power may be one of the main limitations to implement advanced nonlinear control approaches due to high initial investment involved. The cost of nonlinear control prototyping hardware is still very high. To commercialize these advanced techniques, no cheap, portable and capable hardware is available yet. More efficient programming may be required to reduce computational overhead. For instance, in our previous work [89], reinforcement learning based optimal adaptive control for humanoid robots was implemented for a single DOF on dSPACE 1006 employing a sampling time of $1 \mathrm{msec}$. To extend it to two DOF, MATLAB's embedded functions had to be replaced with more efficient $\mathrm{C} / \mathrm{C}++\mathrm{S}$-functions. 
Although actuator saturations usually incur in linear control too, it may, however, be more problematic and catastrophic in case of nonlinear control since the control signal here is very high and aggressive. Hence, it easily leads to windup of the system. In the work by Khan et al. [85], anti-windup compensator was incorporated to deal with actuator saturation issues in the model reference adaptive compliance control of a humanoid robotic arm. However, anti-windup compensators usually degrade the performance of the nominal control scheme.

Nonlinear control schemes may need more expensive actuators to survive longer. Most of the nonlinear controllers pose greater risk to damage expensive systems if not implemented with care. Also, complex and exhaustive tuning process may be involved.

Due to the above mentioned reasons, people in industry are still quite wary of the nonlinear control and there is a big gap between nonlinear control researchers and industrial control engineers. Industries still rely mainly on simple traditional linear schemes. More work is needed to reduce complexity of these advanced nonlinear strategies. Significant efforts are needed to convince the control practitioners to adapt these modern nonlinear techniques.

\section{Conclusion}

In recent years, requirements of modern technology and maturation of various emerging fields have spurred advancements in nonlinear control systems from theoretical as well as applications point of view. Consequently, the topic of nonlinear control has now become a fundamental part of understanding of control engineers and helps them to systematically deal with practical control problems. A common design strategy to solve these problems is to linearize the system valid at a representative operating point(s). This approximate design approach is simple and works sometimes, however, it may impair the original features of nonlinear systems leading to inaccuracy or false conclusions.

The domain of nonlinear control systems has a lively future with numerous interesting and important challenges. The growing applications of nonlinear control systems, such as robots, energy, biology, health care and big data research, are anticipated to further boost up research on advanced theories and development of associated physical technologies.

\section{References}

[1] Ajwad SA, Iqbal J, Ullah MI, Mehmood A. A systematic review of current and emergent manipulator control approaches. Front Mech Eng China. 2015, 10:198-210.

[2] Slotine J-JE, Li W. Applied nonlinear control: Prentice-Hall Englewood Cliffs, NJ, 1991.

[3] Bańos A, Lamnabhi-Lagarrigue F, Montoya FJ. Advances in the control of nonlinear systems: Springer Science \& Business Media, 2001.

[4] Menini L, Zaccarian L, Abdallah CT. Current trends in nonlinear systems and control - In Honor of Petar Kokotovic and Turi Nicosia: Springer, 2006.

[5] Ajwad SA, Ullah M, Khelifa B, Iqbal J. A comprehensive stateof-the-art on control of industrial articulated robots. J Balk Tribol Assoc. 2014, 20:499-521.

[6] Brogan WL. Modern control theory (3rd Edition): Pearson Education India, 2014.

[7] Islam RU, Iqbal J, Khan Q. Design and comparison of two control strategies for multi-DOF articulated robotic arm manipulator. Control Eng Appl Inf. 2014, 16:28-39.

[8] Atherton DP. Early developments in nonlinear control. IEEE Control Syst Mag. 1996, 16:34-43.

[9] Bennett S. A history of control engineering 1800-1930: Peter Peregrinus, Stevenage, UK, 1979.

[10] Bennett S. A history of control engineering 1930-1955: Peter Peregrinus, Stevenage, UK, 1993.

[11] Willems JC. Dissipative dynamical systems. Part I: General theory. Arch Ration Mech Anal. 1972, 45:321-51.

[12] Sontag ED, Wang Y. On characterizations of the input-to-state stability property. Syst Control Lett. 1995, 24:351-9.

[13] Liu Z, Huang D, Xing Y, Zhang C, Wu Z, Ji X. New trends in nonlinear control systems and applications. Abstr Appl Anal. 2015;2015:2.

[14] Isidori A. Nonlinear control systems, Communications and Control Engineering Series. Springer London, UK, 1995.

[15] Kokotović P, Arcak M. Constructive nonlinear control: A historical perspective. Automatica. 2001, 37:637-62.

[16] Poincaré H. Science and hypothesis: Science Press, 1905.

[17] Poincaré $H$. Sur l'équilibre d'une masse fluide animée d'un mouvement de rotation. Acta mathematica. 1885, 7:259-380.

[18] Lyapunov AM. The general problem of motion stability. Annals of Mathematics Studies. 1892, 17.

[19] Duffing G. Erzwungene Schwingungen bei veränderlicher Eigenfrequenz und ihre technische Bedeutung: R, Vieweg \& Sohn, 1918.

[20] Van der Pol B. On "relaxation-oscillations". The London, Edinburgh, and Dublin Philosophical Magazine and Journal of Science. 1926, 2:978-92.

[21] Bode HW. Relations between attenuation and phase in feedback amplifier design. Bell Labs Technical Journal. 1940, 19:421-54.

[22] Krylov N, Bogoliubov N. The application of methods of nonlinear mechanics to the theory of stationary oscillations. Publication. 1934, 8.

[23] Nyquist H. Regeneration theory. Bell Labs Technical Journal. 1932, 11:126-47.

[24] Lur'e A, Postnikov V. On the theory of stability of control systems. Applied mathematics and mechanics. 1944, 8:246-8. 
[25] Emelyanov S. Variable structure control systems. Moscow, Nouka. 1967.

[26] Lorenz EN, Martin P. The essence of chaos. AIP, 1995.

[27] Lorenz E. The butterfly effect. World Scientific Series on Nonlinear Science - Series A. 2000, 39:91-4.

[28] Kalman R, Bertram J. Control system analysis and design via the second method of Lyapunov. Trans ASME. 1960, 1:394400.

[29] Popov V. On absolute stability of non-linear automatic control systems. Automatika i Telemekhanika. 1961, 22:961-79.

[30] Yakubovich VA. The solution of certain matrix inequalities in automatic control theory. Soviet Math Dokl 1962. p. 620-3.

[31] Fuller AT. The early development of control theory. Transactions of the AMSE Journal of Dynamic Systems, Measurement, and Control. 1976, 98:109-18.

[32] Fuller A. The early development of control theory II. Transactions of the AMSE Journal of Dynamic Systems, Measurement, and Control. 1976, 98:224-35.

[33] Luenberger D. An introduction to observers. IEEE Transactions on automatic control. 1971, 16:596-602.

[34] Willems JC. Dissipative dynamical systems part I: General theory. Archive for rational mechanics and analysis. 1972, 45:321-51.

[35] Richalet J, Rault A, Testud J, Papon J. Model predictive heuristic control: Applications to industrial processes. Automatica. 1978, 14:413-28.

[36] Helton JW, James MR. Extending H-infinity control to nonlinear systems. 1999.

[37] Ortega R, Spong MW, Gómez-Estern F, Blankenstein G. Stabilization of a class of underactuated mechanical systems via interconnection and damping assignment. IEEE transactions on automatic control. 2002, 47:1218-33.

[38] Sontag ED, Wang Y. On characterizations of the input-to-state stability property. Systems \& Control Letters. 1995, 24:351-9.

[39] Isidori A. Nonlinear control systems: Springer Science \& Business Media, 2013.

[40] Khan MF, Islam RU, Iqbal J. Control strategies for robotic manipulators. IEEE International Conference on Robotics and Artificial Intelligence (ICRAI)2012. p. 26-33.

[41] Utkin V. Discussion aspects of high-order sliding mode control. IEEE Transactions on Automatic Control. 2016, 61:829-33.

[42] Iqbal J, Ullah MI, Khan AA, Irfan M. Towards sophisticated control of robotic manipulators: An experimental study on a pseudo-industrial arm. Stroj Vestn-J Mech E. 2015, 61:465-70.

[43] Iqbal U, Samad A, Nissa Z, Iqbal J. Embedded control system for AUTAREP - A novel AUTonomous Articulated Robotic Educational Platform. Tehnički vjesnik - Technical Gazette. 2014, 21:1255-61.

[44] Iqbal J, Ajwad SA, Syed ZA, Abdul AK, Islam RU. Automating industrial tasks through mechatronic systems - A review of robotics in industrial perspective. Teh Vjesn. 2016, 23:917-24.

[45] Ullah MI, Ajwad SA, Irfan M, Iqbal J. Non-linear control law for articulated serial manipulators: Simulation augmented with hardware implementation. Elektronika ir Elektrotechnika. 2016, 22:3-7.

[46] Ajwad SA, Iqbal J, Khan AA, Mehmood A. Disturbanceobserver-based robust control of a serial-link robotic manipulator using SMC and PBC techniques. Stud Inform Control. 2015, 24:401-8.
[47] Khan S, Jalani J. Realisation of model reference compliance control of a humanoid robot arm via integral sliding mode control. Mech Sci. 2016, 7:1.

[48] Spiers A, Khan SG, Herrmann G. Biologically inspired control of humanoid robot arms: Robust and adaptive approaches. 1 ed: Springer, 2016.

[49] Iqbal J, Tsagarakis N, Caldwell D. Human hand compatible underactuated exoskeleton robotic system. Electronics Letters. 2014, 50:494-6.

[50] Iqbal J, Khan H, Tsagarakis NG, Caldwell DG. A novel exoskeleton robotic system for hand rehabilitation - Conceptualization to prototyping. Biocybern Biomed Eng. 2014, 34:79-89.

[51] Iqbal J, Tsagarakis N, Caldwell D. Four-fingered lightweight exoskeleton robotic device accommodating different hand sizes. Electron Lett. 2015, 51:888-90.

[52] Muhammad I, Ali K, Iqbal J, Raja AR. Regulation of hypnosis in Propofol anesthesia administration based on non-linear control strategy. Braz J Anesthesiol. 2017, 67:122-30.

[53] Ullah M, Wolkenhauer O. Stochastic approaches for systems biology: Springer Science \& Business Media, 2011.

[54] Dekkers R. Applied systems theory: Springer, 2015.

[55] Barăo M, Lemos JM. Nonlinear control of HIV-1 infection with a singular perturbation model. Biomed Signal Process Control. 2007, 2:248-57.

[56] Shirazian M, Farahi MH. Optimal control strategy for a fully determined HIV model. Intell Control Autom. 2010, 1:15.

[57] Bequette BW. Nonlinear control of chemical processes: A review. Ind Eng Chem Res. 1991, 30:1391-413.

[58] Feng K, Lu J, Chen J. Nonlinear model predictive control based on support vector machine and genetic algorithm. Chinese J Chem Eng. 2015, 23:2048-52.

[59] Gil I, Vargas J, Corriou J. Optimal nonlinear control of an industrial emulsion polymerization reactor. Chem Eng Res Des. 2016, 111:63-82.

[60] Lee JH, Lee JM. Progress and challenges in control of chemical processes. Annu Rev Chem Biomol. 2014, 5:383-404.

[61] Lu Q, Sun Y, Mei S. Nonlinear control systems and power system dynamics: Springer Science \& Business Media; 2013.

[62] Ren Y, Li L, Brindley J, Jiang L. Nonlinear PI control for variable pitch wind turbine. Control Eng Pract. 2016, 50:84-94.

[63] Nazaripouya H, Mehraeen S. Modeling and nonlinear optimal control of weak/islanded grids using FACTS device in a game theoretic approach. IEEE Transactions on Control Systems Technology. 2016, 24:158-71.

[64] Elbanhawi M, Simic M, Jazar R. The efect of receding horizon pure pursuit control on passenger comfort in autonomous vehicles. Intelligent Interactive Multimedia Systems and Services: Springer; 2016. p. 335-45.

[65] Li J, Liu Y, Li C, Chu B. Passivity-based nonlinear excitation control of power systems with structure matrix reassignment. Information. 2013, 4:342-50.

[66] Tang G, Xu Z, Dong H, Xu Q. Sliding mode robust control based active-power modulation of multi-terminal HVDC transmissions. IEEE Transactions on Power Systems. 2016, 31:1614-23.

[67] Mi Y, Fu Y, Li D, Wang C, Loh PC, Wang P. The sliding mode load frequency control for hybrid power system based on disturbance observer. International Journal of Electrical Power \& Energy Systems. 2016, 74:446-52.

[68] lu HH. A review of applications of nonlinear dynamics in power electronics systems. Australian Journal of Electrical and Elec- 
tronics Engineering. 2004, 1:75-81.

[69] Vazquez S, Leon JI, Franquelo LG, Rodriguez J, Young HA, Marquez A, et al. Model predictive control: A review of its applications in power electronics. IEEE Industrial Electronics Magazine. 2014, 8:16-31.

[70] Guzman R, de Vicuna LG, Morales J, Castilla M, Matas J. Sliding-mode control for a three-phase unity power factor rectifier operating at fixed switching frequency. IEEE Transactions on Power Electronics. 2016, 31:758-69.

[71] Huangfu Y, Zhuo S, Rathore AK, Breaz E, Nahid-Mobarakeh B, Gao F. Super-twisting differentiator-based high order sliding mode voltage control design for DC-DC buck converters. Energies. 2016, 9:494.

[72] Sahara A, Kessal A, Rahmani L, Gaubert J-P. Improved sliding mode controller for shunt active power filter. ournal of Electrical Engineering and Technology. 2016, 11:662-9.

[73] Salehifar M, Arashloo RS, Moreno-Eguilaz M, Sala V, Romeral L. Observer-based open transistor fault diagnosis and faulttolerant control of five-phase permanent magnet motor drive for application in electric vehicles. IET Power Electronics. 2015, 8:76-87.

[74] Pradeep DJ, Noel MM, Arun N. Nonlinear control of a boost converter using a robust regression based reinforcement learning algorithm. Engineering Applications of Artificial Intelligence. 2016, 52:1-9.

[75] Konstantopoulos GC, Zhong Q-C. Nonlinear control of singlephase PWM rectifiers with inherent current-limiting capability. 2016.

[76] Yang L, Zhang W, Zhang X, Li G. Nonlinear variable frequency control of high power switched-capacitor converter. 2016 IEEE 8th International Power Electronics and Motion Control Conference (IPEMC-ECCE Asia): IEEE; 2016. p. 3472-6.

[77] Sun F, Huang X, Rudolph J, Lolenko K. Vehicle state estimation for anti-lock control with nonlinear observer. Control Eng Pract. 2015, 43:69-84.

[78] Hervas JR, Reyhanoglu M, Tang H, Kayacan E. Nonlinear control of fixed-wing UAVs in presence of stochastic winds. Commun Nonlinear Sci. 2016, 33:57-69.
[79] Mahapatra S, Subudhi B, Rout R, Kumar BK. Nonlinear H? control for an autonomous underwater vehicle in the vertical plane. 4th IFAC Conference on Advances in Control and Optimization of Dynamical Systems. India2016. p. 391-5.

[80] Ansarifar G. Control of the nuclear steam generators using adaptive dynamic sliding mode method based on the nonlinear model. Ann Nucl Energy. 2016, 88:280-300.

[81] Song Y, Wang X, Teng H, Guan Y. Nonlinear parametric predictive control for the temperature control of bench-scale batch reactor. Appl Therm Eng. 2016, 102:134-43.

[82] Schirrer A, Brandstetter M, Leobner I, Hauer S, Kozek M. Nonlinear model predictive control for a heating and cooling system of a low-energy office building. Energ Buildings. 2016, 125:86-98.

[83] Yang J, Zhang G, Ma K. A nonlinear control method for pricebased demand response program in smart grid. International Journal of Electrical Power \& Energy Systems. 2016, 74:322-8.

[84] Black WS, Haghi P, Ariyur KB. Adaptive systems: History, techniques, problems, and perspectives. Systems. 2014, 2:60660.

[85] Khan SG, Herrmann G, Pipe T, Melhuish C, Spiers A. Safe adaptive compliance control of a humanoid robotic arm with antiwindup compensation and posture control. Int J Soc Robot. 2010, 2:305-19.

[86] Lewis FL, Dawson DM, Abdallah CT. Robot manipulator control: Theory and practice: CRC Press, 2003.

[87] Sutton RS, Barto AG, Williams RJ. Reinforcement learning is direct adaptive optimal control. IEEE Control Systems. 1992 12:19-22.

[88] Vrabie D, Vamvoudakis KG, Lewis FL. Optimal adaptive control and differential games by reinforcement learning principles: IET, 2013.

[89] Khan SG, Herrmann G, Lewis FL, Pipe T, Melhuish C. Reinforcement learning and optimal adaptive control: An overview and implementation examples. Annu Rev Control. 2012, 36:42-59. 